\title{
Nuclear and Hadronic Physics at Large $N_{c}$
}

\section{Thomas D. Cohen ${ }^{* \dagger}$}

University of Maryland, College Park

E-mail: cohen@umd. edu

This talk briefly surveys some of the advances in large $N_{c}$ QCD in nuclear and hadronic physics during the past decade or so. It highlights recent results including an analytic result for the total nucleon-nucleon cross section in nucleon-nucleon scattering in the extreme large $N_{c}$ limit $\left.\left(\log N_{c} \gg 1\right)\right)$; it is given by $\sigma^{\text {total }}=\frac{2 \pi \log ^{2}\left(N_{c}\right)}{m_{\pi}^{2}}$ for $\sqrt{s}$ much larger than the QCD scale. The talk also stresses a demonstration that given certain technical assumptions, it can be shown that QCD must have a Hagedorn spectrum (i.e., a spectrum where the number of hadrons grows exponentially with hadron mass). Another theme in the talk is the phenomenology of a variant of the large $N_{c}$ limit based on quarks in the two-index antisymmetric representation. This variant of large $N_{c}$ QCD is qualitatively different from the conventional one in which quarks are in the fundamental representation and corresponds to a distinct large $N_{c}$ limit with a distinct $1 / N_{c}$ expansion. The description of nuclear forces and nuclear matter in both variants of the large $N_{c}$ limit are discussed.

Sixth International Conference on Quarks and Nuclear Physics

April 16-20, 2012

Ecole Polytechnique, Palaiseau, Paris

\footnotetext{
* Speaker.

${ }^{\dagger}$ The support of the United States Department of Energy is gratefully acknowledged.
} 


\section{Introduction}

The talk reviews some of the advances during the last decade or so. I will assume basic familiarity with the basic idea of large $N_{c}$ analysis and will not review the classic analysis of 't Hooft[1] and Witten[2]. It is not intended to completely cover the landscape of the subject which is very large. Rather it will idiosyncratically address a few of the themes which I find particularly interesting and illustrate these with a few examples. The topic is very broad. Clearly, given the limitations of time in the talk, I will quickly touch on many issues and refer the reader to the original literature for more details. Indeed, the talk is in the spirit of Art Buchwald's classic humor column which describes how Peter Stone, an American tourist, first broke the six-minute Louvre in 1950 by skipping almost everything and racing past a few masterpieces[3]. While I cannot assert that topics discussed in this talk are scientific masterpieces, it remains true that the very rapid discussion does not do them justice.

Rather than attempting to introduce the topics in the talk in a careful and coherent way, let me just present an overview of the topics and how they are connected in fig. 1.

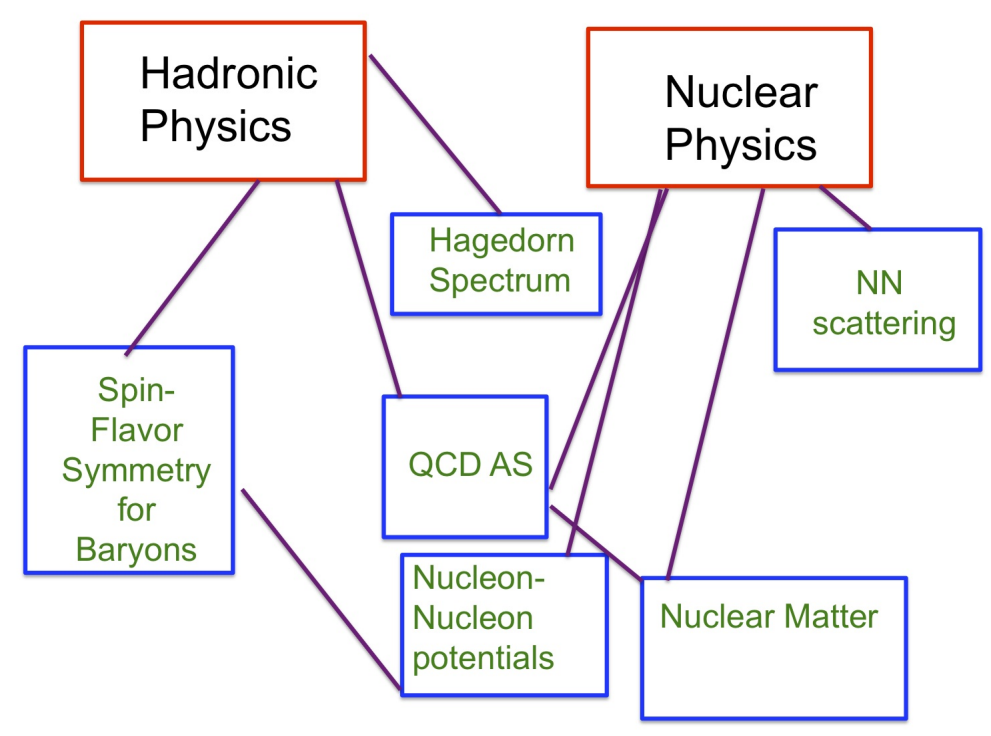

Figure 1: An overview of the talk.

\section{The Nucleon-Nucleon Total Cross Section}

The first result that I wish to discuss is the nucleon-nucleon total cross section at large $N_{c}$, which is given by [4]

$$
\sigma^{\text {total }}=\frac{2 \pi \log ^{2}\left(N_{c}\right)}{m_{\pi}^{2}}
$$

which holds for $s \gg \Lambda_{\mathrm{QCD}}$ and in the extreme large $N_{c}$ limit in which $\log \left(N_{c}\right) \gg 1$. The result is remarkable in that, unlike virtually all results obtained previously in large $N_{c} \mathrm{QCD}$, it is an 
analytic expression for an observable rather than a statement of an observable scale. Note that the prediction is that the total cross section is independent of momentum in this regime-which to good approximation it is. However, the predicted cross section is roughly a factor of 4 above the empirical one in the few $\mathrm{GeV}$ range. This is presumably a consequence of the fact that the result only holds at extreme $N_{c}$. The structure which involves the square of the logarithm is reminiscent of the Froissart-Martin bound[5]. As discussed briefly in ref. [4], this may not be entirely coincidental.

\section{The Hagedorn Spectrum}

A Hagedorn spectrum is one in which the number of hadrons grows exponentially with the mass of the hadron at large mass. It was postulated in pre-QCD days[6]. The idea is problematic in QCD: hadrons are resonances and the widths add ambiguity to the mass of hadrons, particularly at large mass. However, at large $N_{c}$ the widths of mesons go to zero and the idea of a Hagedorn spectrum is well posed. Recently[7] a theoretical argument has been developed that QCD must have a Hagedorn spectrum at large $N_{c}$ provided that certain technical assumptions are true.

The theoretical inputs to this are confinement (in the sense that only color neutral physical states exist), asymptotic freedom, standard assumptions about regime of validity of perturbation theory (namely that perturbation theory is valid when perturbation corrections are small), and some plausible technical assumptions about the ordering of limits. It should be stressed that because of these assumptions, it is not a rigorous theorem in the mathematical sense.

The approach is based on a matrix of Euclidean space correlation functions from some set of single-color-trace operators with fixed quantum numbers. The derivative of the trace of the logarithm of this matrix with respect to Euclidean time can be shown to bound the sum of the lightest $d$ mesons where $d$ is the dimension of the matrix. There are two keys to the approach. The first is that the number of local single color trace operators of fixed quantum number grows exponentially with the mass dimension of the operator. While each operator does not create a distinct hadron, this exponential growth in the number of operators is ultimately translated into an exponential growth with mass in the number of hadrons. The second is that the regime of validity of perturbation theory for the trace of the $\log$ of a matrix of point-to-point correlators is independent of the dimension of the operator at large $N_{c}$. (Note this depends on the planarity of diagrams at large $N_{c}$ and is false for finite $N_{c}$ ).

The details of the derivation are too involved to discuss in the limited time, so I will refer you to the original literature. Let me note, though, that it is quite satisfying that given some very natural assumptions a Hagedorn spectrum will automatically arise at large $N_{c}$.

\section{QCD and Its Large $N_{C}$ Limits}

The large $N_{c}$ limit of QCD is not unique. For gluons there is a unique prescription for how to extrapolate $\mathrm{SU}(30) \rightarrow \mathrm{SU}\left(N_{c}\right)$. However, for quarks we can choose different representations of the gauge group. Asymptotic freedom restricts the possibilities to the fundamental (F), adjoint (Adj), two index symmetric (S), and two-index anti-symmetric (AS). Adj transforms like gluons (traceless fundamental color-anticolor) and the dimension of the representation is $N_{c}^{2}-1$ which is 8 for $N_{c}=3$. S transforms like two colors (eg., fundamental quarks) with indices symmetrized; 
the dimension $N_{c}^{2}-N_{c}$ which is 6 for $N_{c}=3$. At $N_{c}=3$ neither of these is the QCD of our world. Note, however, that AS transforms like two colors (eg., fundamental quarks) with indices antisymmetrized and the dimension of the representations is $\frac{1}{2} N_{c}\left(N_{c}-1\right)$ which is 3 for $N_{c}=3$. Indeed, it is easy to see that at $N_{c}=3$, quarks in the AS representation are indistinguishable from the (anti- )fundamental. However, quarks in the AS and F extrapolate to large $N_{c}$ in different ways. The large $N_{c}$ limits are physically different. The $1 / N_{c}$ expansions are different. Moreover, a priori it is not obvious which expansion is better; it may well depend on the observable in question.

While the idea of QCD(AS) is old[8], it was not seriously explored initially-in contrast to $\mathrm{AQCD}(\mathrm{F})$ which has been studied intensively for 40 years. Thus it is probably a good idea to follow the advice of American poet, Robert Frost, and take the road less travelled. The idea of QCD(AS) was revived in the early part of this decade by Armoni, Shifman and Veneziano who discovered a remarkable duality that emerges at large $N_{c}$ between QCD(AS) and QCD(Adj)[9]. While interesting theoretically, the duality may not be phenomenologically useful directly. However, the general question of the phenomenology of QCD(AS) at large $N_{c}$ remains of interest.

In both $\mathrm{QCD}(\mathrm{F})$ and $\mathrm{QCD}(\mathrm{AS})$ meson-meson interactions are weak, but they are systematically smaller for $\mathrm{QCD}(\mathrm{AS})$. The n-meson vertex in the two cases scale as

$$
g^{\mathrm{n}-\text { meson }} \sim N_{c}^{1-n / 2} \text { for } \mathrm{QCD}(\mathrm{F}), \quad g^{\mathrm{n}-\text { meson }} N_{c}^{2-n} \text { for } \mathrm{QCD}(\mathrm{AS})
$$

The critical difference between the two approaches is that while both approaches involve planar diagrams at leading order, quark loops are not suppressed in QCD(AS). Whether this is a bug or a feature depends on circumstance. Thus, the OZI rule is not automatically enforced in QCD(AS) at large $N_{c}$ as it is in $\mathrm{QCD}(\mathrm{F})$. To the extent that the OZI rule holds, one must regard the inclusion of internal quark loops as a bug. However, there are cases of large OZI violation (such as the $\eta^{\prime}-\pi$ mass difference) or other phenomena involving the anomaly in which it should be regarded as a feature.

In both $\mathrm{QCD}(\mathrm{F})$ and $\mathrm{QCD}(\mathrm{AS})$ baryons are heavy, scaling as $N_{c}[10]$ and $N_{c}^{2}$ [2], respectively. The consistent treatment of baryons in QCD (AS) turns out to be rather subtle. One cannot simply borrow Witten's combinatorial arguments from $\mathrm{QCD}(\mathrm{F})$. The combinatorics for $\mathrm{QCD}(\mathrm{AS})$ turn out to be far more intricate, and to proceed, new tools need to be developed. However, this has been done and the upshot is that they scale as $N_{c}^{2}$ as one might expect: a baryon contains a quark of each color type and this number scales as $N_{c}^{2}$.

Generic meson-baryon coupling is strong in both large $N_{c}$ limits but stronger for QCD(AS): for QCD(F), $g_{N m} \sim N_{c}^{\frac{1}{2}}$, while QCD(AS) $g_{N m} \sim N_{c}$.

It has long been known that the strong meson-baryon coupling constant in $\mathrm{QCD}(\mathrm{F})$ implied the existence of an emergent $\mathrm{SU}\left(2 N_{F}\right)$ spin-flavor symmetry at large $N_{c}$ [11]. The argument goes through for $\mathrm{QCD}(\mathrm{AS})$; the only difference being the scale of the symmetry breaking scales as $N_{c}^{2}$ rather than $N_{c}$. Such a symmetry implies that there is an infinite tower of baryon states with I=J which are degenerate at large $N_{c}$ and with relative matrix elements fixed by Clebsch-Gordan coefficients of the group. For $N_{c}=3$ the nucleon and $\Delta$ are identified as members of the band. (Other states are large $N_{c}$ artifacts). The $N-\Delta$ mass splitting scales as $1 / N_{c}$ and $1 / N_{c}^{2}$ for QCD(F) and QCD(AS), respectively.

The existence of this emergent symmetry and knowledge of the scaling of its breaking allows one to derive mass relations among the octet and decuplet baryons which hold to various orders 
of accuracy in SU(3) flavor breaking and $1 / N_{c}$. These relations were first derived in the $1990 \mathrm{~s}$ for the case $\mathrm{QCD}(\mathrm{F})$ and the relations held much better than an analogous relations based solely on SU(3) flavor[12]; the $1 / N_{c}$ scaling had real predictive power. One might guess that if one used QCD(AS), which has a different $N_{c}$ counting, it would not do as well. Remarkably this is not the case, as discussed in refs.[13]. The mass relations in both $\mathrm{QCD}(\mathrm{F})$ and $\mathrm{QCD}(\mathrm{AS})$ hold much better empirically than pure SU(3) flavor. Ultimately, the reason that they can both do well is that for $N_{c}=3$ there is not quite enough dynamic range to clearly distinguish the two.

\section{Nucleon-Nucleon Interactions}

It is by no means obvious that large $N_{c}$ analysis is likely to be as useful in nuclear physics as it has proven in hadronic physics. The reason it may prove to be problematic phenomenologically is due to small scales which arise in nuclear physics; these are often much smaller than the $\sim 1$ $\mathrm{GeV}$ scale which typically characterizes hadronic physics. For example, the binding energy per nucleon is only about $16 \mathrm{MeV}$ and the Fermi momentum about $279 \mathrm{MeV}$. Moreover these light scales do not arise for reasons associated with large $N_{c}$; the Fermi momentum is of order $N_{c}^{0}$ and the binding energy is of order $N_{c}^{1}$. This means that the ordering of scales seen in the large $N_{c}$ world is not present in the $N_{c}=3$ world. It is highly plausible that for most nuclear observables, $N_{c}=3$ is outside of the radius of convergence of the $1 / N_{c}$ approximation. This does not mean the large $N_{c}$ limit is necessarily without interest—however, in many cases this interest may be of a theoretical nature.

One place where large $N_{c}$ counting appears to give insight into the world of $N_{c}=3$ is for the nucleon-nucleon potential. The generic scaling of this is easy to deduce-at leading order the potential scales as $N_{c}$ for $\mathrm{QCD}(\mathrm{F})$ and $N_{c}^{2}$ for $\mathrm{QCD}(\mathrm{AS})$. What is more interesting is the pattern of sizes of the various angular momentum and isospin combinations which contribute to the potential. One can use the emergent spin-flavor symmetry to deduce the relative sizes of the various contributions; moreover, the pattern obtained for these is in qualitative agreement for what is observed in phenomenological potentials[14].

It is noteworthy that these patterns are precisely what would be obtained with one-mesonexchange-potentials if the meson-baryon couplings follow the $N_{c}$ scalings of the emergent spinflavor symmetry[15]. Naively, if one looks at contributions to the potential from two-meson exchange the scaling rules break down since the leading contribution to the potential from the Feynman diagrams scales as $N_{c}$ for QCD(F) (and $N_{c}^{2}$ for QCD(AS)). However, it can be shown that when the emergent spin-flavor symmetry is taken into account, there exist cancellations[15] of all terms which violate the scaling rules of ref. [14]. It turns out that three-meson-exchange is subtle and that direct computation of the potential from the diagrams violates the scaling rules, even when cancelations are included. However, the potential derived this way is energy-dependent; when transformed to an energy-independent potential the scaling rules are restored[16].

The preceding results on potentials are interesting. However, it is worth recalling that potentials are not physical observables. Moreover, as noted by Witten[2], nucleon-nucleon scattering does not have a smooth large $N_{c}$ limit at fixed momentum; rather, one must study the regime with fixed velocity. However, this regime cannot be described by a potential model since it is well above the meson-production threshold. How can one encode the fact that nucleon-nucleon interactions 
are strong in this regime? The natural way is via the logarithm of the two-nucleon S-matrix:

$$
\log (S) \sim N_{c}
$$

where the result is given for $\mathrm{QCD}(\mathrm{F})$. Equation (2.1) describing the $\mathrm{NN}$ total cross section is derived from Eq. (5.1).

\section{Nuclear Matter}

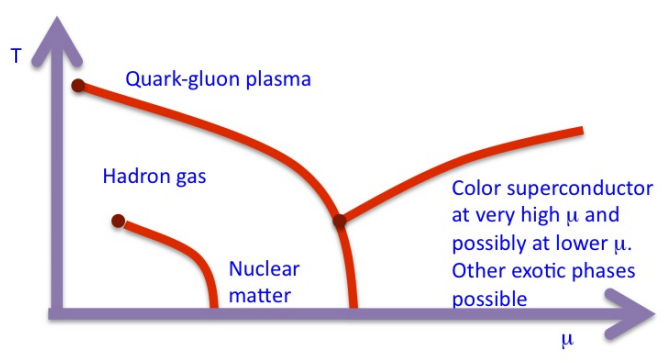

A

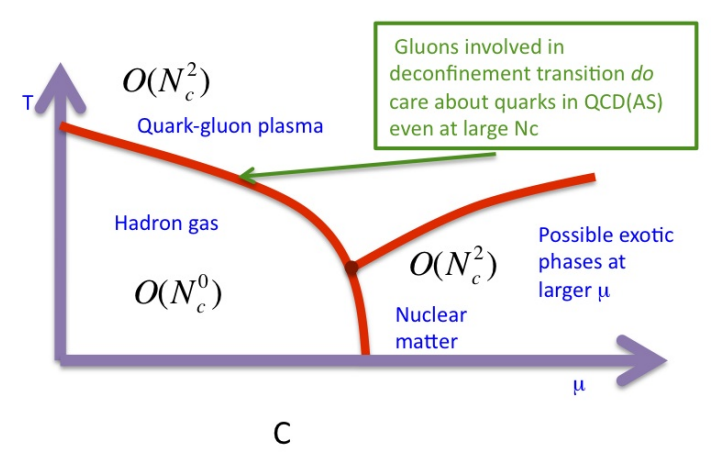

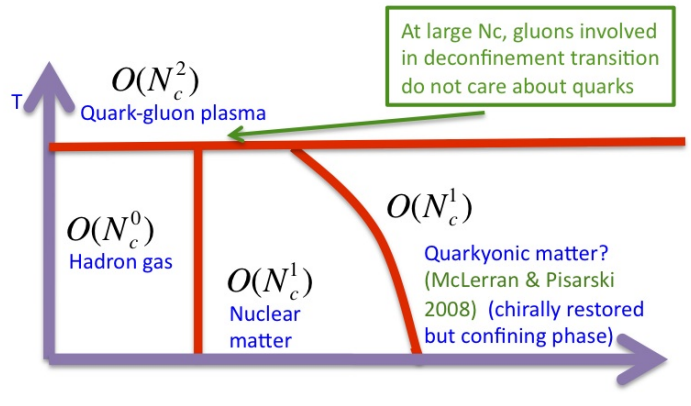

B

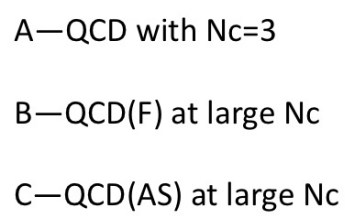

$\mathrm{A}-\mathrm{QCD}$ with $\mathrm{Nc}=3$

$\mathrm{B}-\mathrm{QCD}(\mathrm{F})$ at large $\mathrm{NC}$

$\mathrm{C}-\mathrm{QCD}(\mathrm{AS})$ at large Nc

Figure 2: Cartoons of the phase diagram for A. QCD with $N_{c}=3$, B. QCD(F) at large $N_{c}$ and C. QCD(AS) at large $N_{c}$.

Cold nuclear matter is crystalline and saturates in both large $N_{c}$ limits. The saturation density and binding energy per nucleon scale as:

$$
\mathrm{QCD}(\mathrm{F}): \quad \rho_{\mathrm{sat}} \sim N_{c}^{0} B / A \sim N_{c}^{1} \quad \mathrm{QCD}(\mathrm{AS}): \rho_{\mathrm{sat}} \sim N_{c}^{0} B / A \sim N_{c}^{2} .
$$

It is easy to understand why. Pion exchange is the dominant long-range interaction and has an attractive channel. Any attractive quantum system with parametrically strong forces or heavy mass will become arbitrarily well localized around the classical minimum yielding a deeply bound crystal. 
However, while both limits are similar in this respect, when temperature is included, their equations of state are expected to be qualitatively different both from each other and from QCD at $N_{c}=3$. Consider the regime where the temperature and the quark chemical potential are both of order $N_{c}^{0}$. In fig. 2 cartoon versions of the expected phase diagrams are given. Two things are key to understanding these. The first is that at $N_{c}=3$ there are expected to be critical points representing the endpoints of lines of first-order transitions. These disappear in both versions of the large $N_{c}$ limit since the regions they separate scale differently with $N_{c}$ and cannot be smoothly connected at large $N_{c}$. A second key difference is that the critical temperature of $\mathrm{QCD}(\mathrm{F})$ at large $N_{c}$ has no dependence on the chemical potential. This is because quark contributions to the energy are subleading in this limit. One important lesson of this is that large $N_{c}$ analysis is clearly not appropriate to discern the nature of the phase diagram. It is noteworthy, however, that in at least one important way the phase diagram of QCD(AS) is closer to the diagram of the real world than is $\mathrm{QCD}(\mathrm{F})$.

What about in the regime of cold nuclear matter at large $\mu$ ? It is generally expected that QCD with $N_{c}=3$ at high $\mu$ is in a color superconducting phase[17], although in principle it is possible that some heretofore unknown phase is lower. In the case of QCD(F) at large $N_{c}$, this is known not to happen: while a Fermi gas at large $\mu$ has a BCS instability towards a superconducting phase, there is a stronger instability[18] towards an inhomogeneous phase of the Deryagin, Grigoriev, and Rubakov (DGR) type[19]. The BCS instability is suppressed scaling as $e^{-N_{c}}$ since the condensate is not a color singlet. In contrast, the DGR condensate is a color singlet (though not gauge invariant) and does not have this suppression. Thus, if the DGR instability occurs, which it does at extremely large $N_{c}$ in $\mathrm{QCD}(\mathrm{F})$, it will dominate. Since this only occurs at very high $N_{c}$, it does not occur in the physical world at $N_{c}=3$. However, an RG analysis shows that in QCD(AS) the DGR instability does not occur[20] while the BCS instability does. Thus, it is plausible that QCD(AS) is qualitatively similar to QCD at $N_{c}=3$ in having as its ground state a color superconductor[20].

\section{References}

[1] G. 't Hooft, Nucl. Phys. B 72 (1974) 46; G. 't Hooft, Nucl. Phys. B 75 (1974) 461.

[2] E. Witten, Nucl. Phys. B 160 (1979) 57.

[3] The Saturday Evening Post, October 1, 1984.

[4] arXiv:1203.5843.

[5] M. Froissart, Phys. Rev. 123 (1961) 1053; A. Martin, Phys. Rev. 129 (1963) 1432.

[6] Hagedorn R., Nov. Cimen. Suppl. 3 (1965) 147; Hagedorn R., Nov. Cimen. 56A (1968) 1027

[7] T. D. Cohen, JHEP 06 (2010) 098; T. D. Cohen and V. Krejcicik, JHEP 08 (2011) 138.

[8] E. Corrigan and P. Ramond, Phys. Lett. B 87 (1979) 73.

[9] A. Armoni, M. Shifman and G. Veneziano, Nucl. Phys. B 667 (2003) 170: Phys. Rev. Lett. 91 191601 (2003) 191601.

[10] S. Bolognesi, Phys. Rev. D 75 (2007) 065030; A. Cherman and T. D. Cohen, JHEP 0612 (2006) 035; T. D. Cohen, D. L. Shafer and R. F. Lebed, Phys. Rev. D81 (2010) 036006. 
[11] J. L. Gervais and B. Sakita, Phys. Rev. Lett. 52 (1984) 87; Phys. Rev. D 30 (1984) 1795; R. F. Dashen and A. V. Manohar, Phys. Lett. B 315, (1993) 425; B 315 (1993) 438; R. F. Dashen, E. E. Jenkins and A. V. Manohar, Phys. Rev. D 49 (1994) 4713; Phys. Rev. D 51 (1995) 3697.

[12] E. E. Jenkins and R. F. Lebed, Phys. Rev. D 52 1995) 282.

[13] A. Cherman, T.D. Cohen and R.F. Lebed, Phys. Rev. D 80 (2009) 036002; arXiv:1205.1009.

[14] D. B. Kaplan and M. J. Savage, Phys. Lett. B 365 (1996) 244; D. B. Kaplan and A. V. Manohar, Phys. Rev. C 56 (1997) 76.

[15] M. K. Banerjee, T. D. Cohen and B. A. Gelman, Phys. Rev. C 65 (2002) 034011.

[16] A. V. Belitsky and T. D. Cohen, Phys. Rev. C 65 (2002) 064008; T. D. Cohen, Phys. Rev. C 66 (2002) 064003.

[17] M. G. Alford, A. Schmitt, K. Rajagopal and T. Schafer, Rev. Mod. Phys. 80 (2008) 1455 .

[18] E. Shuster and D. T. Son, Nucl. Phys. B 573 (2000) 434.

[19] D. V. Deryagin, D. Y. Grigoriev and V. A. Rubakov, Int. J. Mod. Phys. A 7 (1992) 659.

[20] M. I. Buchoff, A. Cherman, T.D. Cohen Phys. Rev. D81 (2010) 125021. 